\title{
Serum Levels of Brain-Derived Neurotrophic Factor at 4 Weeks and Response to Treatment with SSRIs
}

\author{
Reiji Yoshimura $^{1 凶}{ }^{\bowtie}$, Taro Kishi ${ }^{2,3}$, Hikaru Hori', Asuka Katsuki ${ }^{1}$, Atsuko Sugita-Ikenouchi ${ }^{1}$, \\ Wakako Umene-Nakano', Kiyokazu Atake', Nakao Iwata ${ }^{3}$, and Jun Nakamura' \\ ${ }^{1}$ Department of Psychiatry, University of Occupational and Environmental Health, Kitakyushu, Japan \\ 'Zucker Hillside Hospital, Glen Oaks, New York, USA \\ ${ }^{3}$ Fujita Health University School of Medicine, Toyoake, Japan
}

\begin{abstract}
Objective It is important to predict a response to an antidepressant in early time after starting the antidepressant. We previously reported that serum brain-derived neurotrophic factor (BDNF) levels in responders to treatment with antidepressants were increased, whereas, those in nonresponders were not. Therefore, we hypothesized that the changes in serum levels of BDNF from baseline (T0) to 4 weeks (T4) after treatment with selective serotonin reuptake inhibitors (SSRIs) predict the response to the treatment at 8 weeks (T8) in depressed patients. To confirm the hypothesis, we measured serum BDNF at T0, T4, and T8 during the treatment with SSRIs (paroxetine, sertraline, and fluvoxamine).
\end{abstract}

Methods One hundred fifty patients (M/F; 51/99, age; 50.4 15.1 years) met major depressive disorder (MDD) using by DSM-IV-TR enrolled in the present study. We measured serum BDNF concentrations at T0, T4, and T8 in patients with MDD treated with SSRIs.

Results The changes in serum BDNF, age, sex, dose of SSRIs, and HAMD-17 score did not predict the response to SSRIs at T8.

Conclusion These results suggest that the changes in serum BDNF levels from T0 to T4 could not predict the subsequent responses to SSRIs at T8.

Psychiatry Investig 2014;11:84-88

Key Words Brain-derived neurotrophic factor, Serum, Depression, Response, Selective serotonin reuptake inhibitor.

\section{INTRODUCTION}

Treatment of depression with currently available antidepressants is not satisfied, because only $30-40 \%$ of real-life patients with major depressive disorder reach a remission with the first selected antidepressant. ${ }^{1}$ Therefore, it is important to develop new strategies to increase remission rates and to shorten the time to remission. Prediction of response to antidepressants at early period after starting the antidepressant is necessary to decide continuing the antidepressant, changing other one, or augmentation strategy. A substantial body of evidence from many retrospective studies shows that non-improvement, often defined as $<20 \%$ reduction of depressive symp-

Received: January 18, 2013 Revised: April 15, 2013

Accepted: June 14, 2013 Available online: January 21, 2014

$\triangle$ Correspondence: Reiji Yoshimura, MD, PhD

Department of Psychiatry, University of Occupational and Environmental Health, 1-1 Iseigaoka, Yahatanishi-ku, Kitakyushu 8078555, Fukuoka, Japan Tel: +81936917253, Fax $:+81936924894$

E-mail: yoshi621@med.uoeh-u.ac.jp

(a) This is an Open Access article distributed under the terms of the Creative Commons Attribution Non-Commercial License (http://creativecommons.org/licenses/by$\mathrm{nc} / 3.0$ ) which permits unrestricted non-commercial use, distribution, and reproduction in any medium, provided the original work is properly cited. toms assessed with a routine scale like the Hamilton Rating Scale for Depression (HAMD) ${ }^{2}$ after 14 days of treatment is a highly specific marker for final treatment failure with an unchanged treatment. ${ }^{3}$

Brain-derived neurotrophic factor (BDNF) plays an important role in the pathophysiology of depression. ${ }^{4,5}$ In addition, blood (serum or plasma) levels of BDNF is a biomarker for depressive state. ${ }^{6-9}$ We previously reported that baseline serum BDNF levels predict subsequent response to mirtazapine in Japanese patients with major depressive disorder..$^{10}$ In another study, we demonstrated that baseline serum BDNF levels did not significantly different between responder and non-responders to selective serotonin reuptake inhibitor (SSRI) or serotonin noradrenaline reuptake inhibitor medications. ${ }^{11,12}$ Wolkowitz et al. ${ }^{13}$ reported that pre-treatment serum BDNF levels were significantly higher in the responders than in the non-responders. From these findings into account, it is controversial baseline serum BDNF levels could predict later response to treatment with antidepressants. Recently, Tadic et al. ${ }^{14}$ reported that the non-increase of serum BDNF level and the non-improvement of HAMD from baseline to day 7 or 14 
predicted later non-response and non-remission with moderate to high specificity. In clinical practice, prediction for response to antidepressants within an early point is an important issue. Therefore, we hypothesized that serum levels 4 weeks after starting SSRIs could predict subsequent response to SSRIs, it could help to decide in changing other antidepressants, increase the same antidepressant, or choosing augmentation strategy.

We consider that if changes in serum BDNF levels at early point predict the subsequent outcome of the response to SSRIs, the changes in serum BDNF levels might become useful tool for predictions for SSRIs response. To confirm the hypothesis, we examined the relation between serum BDNF levels at 4 weeks and subsequent clinical response to SSRIs.

\section{METHODS}

This study included 150 in- or outpatients in our university hospital who met the DSM-IV-TR criteria ${ }^{15}$ for major depressive disorder without psychotic features and who scored at least 16 on the 17-items of the Hamilton Raring Scale for Depression (HAMD-17). Fifty-one patients were male and 99 were female. The age of the subjects ranged from 22 to 76 years (mean $\pm S D=50.4 \pm 15.1$ ). None had received any antidepressant drugs or mood stabilizers at least two weeks prior to the study. All patients were physically healthy and no subjects had a history of alcohol and/or drug abuse. None had co-morbid any anxiety disorders and personality disorders. The patients were treated with paroxetine, sertraline, or fluvoxamine. There were 60 cases treated with paroxetine, 72 cases treated with sertraline, and 28 cases treated with fluvoxamine. Initial dose of paroxetine, sertraline, or fluvoxamine were $10 \mathrm{mg} /$ day, $25 \mathrm{mg} /$ day, or $25 \mathrm{mg} /$ day, respectively, and the dose was increased until the patients tolerated adverse effects (the dose was not fixed). Maximum dose of paroxetine, sertraline or fluvoxamine were $40 \mathrm{mg} /$ day, $100 \mathrm{mg} /$ day, or $150 \mathrm{mg} /$ day, respectively. The average dose of each antidepressant was as follows; paroxetine: $30.5 \pm 12.4 \mathrm{mg} /$ day, sertraline: $76.7 \pm 24.0 \mathrm{mg} /$ day, fluvoxamine: $100 \pm 26.3 \mathrm{mg} /$ day. We evaluated the severity of depressive state using the HAMD-17 before (T0) and 4 (T4) and 8 weeks (T8) after treatment with paroxetine, sertraline, or fluvoxamine. We defined the patients whose scores of the HAMD-17 decreased $50 \%$ or more as responders, whereas, below $50 \%$ as nonresponders.

All blood samples were taken at 7:00 am before breakfast (at least 12 hours after the last medication) before (T0) and 4 (T4) and 8 weeks (T8) after treatment with paroxetine, sertraline, or fluvoxamine. Fifteen milliliters of venous blood was drawn with the patient in the supine position, after the patient had been lying at rest overnight. The serum samples were quickly separated in a centrifuge $\left(2000 \mathrm{~g}, 10 \mathrm{~min}, 4^{\circ} \mathrm{C}\right)$ and stored at $-80^{\circ} \mathrm{C}$ until assay. The serum BDNF levels were measured using a BDNF Emax Immunoassay Kit (Promega, Madison, WI, USA) according to the manufacturer's instructions. In short, 96-well microplates were coated with anti-BDNF monoclonal antibody and incubated at $4^{\circ} \mathrm{C}$ for 18 hours. The plates were incubated in a blocking buffer for 1 hour at room temperature. The samples diluted with assay buffer by 100 -times and BDNF standards were kept at room temperature under conditions of horizontal shaking for 2 hours, followed by washing with the appropriate washing buffer. The plates were incubated with antihuman BDNF polyclonal antibody at room temperature for 2 hours and washed with the washing buffer. The plates were then incubated with anti-IgY antibody conjugated to horseradish peroxidase for 1 hour at room temperature, and incubated in peroxidase substrate and tetramethylbenzidine solution to induce a color reaction. The reaction was stopped with $1 \mathrm{~mol} / \mathrm{L}$ hydrochloric acid. The absorbance at $450 \mathrm{~nm}$ was measured with an Emax automated microplate reader. Measurements were performed in duplicates. The standard curve was linear from $5 \mathrm{pg} / \mathrm{mL}$ to $5000 \mathrm{pg} / \mathrm{mL}$, and the detection limit was $10 \mathrm{pg} / \mathrm{mL}$. Cross-reactivity to related neurotrophins (NT-3, NT-4, NGF) was less than 3\%. Intra- and interassay coefficients of variation were about $5 \%$ and $7 \%$, respectively. The recovery rate of the exogenous added BDNF in the measured plasma samples was more than $95 \%$.

Actually, we examined the change of serum BDNF levels in same samples in a same day at time 8:00, 12:00, 16:00, 20:00, 24:00, and no significant changes in serum BDNF were observed within a day (data not shown). This study was approved by the ethics committee of the University of Occupational and Environmental Health, and written informed consent was obtained from all participants.

\section{Statistical analysis}

The logistic regression analysis was performed for the response to SSRIs at T4 as a dependent variable and changes in BDNF, sex, age, dose of SSRIs at T8, and HAMD-17 at T0 as independent variables. The area under the curve (ROC-AUC) was made for the prediction of the response at T8 with using a BDNF change from T0 to T4. We used the repeated measures of ANOVA comparing HAMD-17 scores and serum BDNF levels at each point. Significance was set at $<0.05$. All analyses were done using the Japanese version of SPSS v15.1 (SPSS, Tokyo, Japan).

\section{RESULTS}

Among 150 patients, 70 (46.3\%) were responders to treat of SSRIs at week 8 , and the rest (53.3\%) were nonresponders. 
Table 1. Independent factors regarding response to SSRIs

\begin{tabular}{|c|c|c|c|c|}
\hline Dependent variable & Independent variable & Wald chi-square & $\mathrm{p}$ & Adjusted $\mathrm{R}^{2}$ \\
\hline \multirow{5}{*}{ Responder/Nonresponder } & Change in BDNF (T4-T0)/T0 & 0.522 & 0.470 & \multirow{5}{*}{0.0218} \\
\hline & Sex & 0.727 & 0.394 & \\
\hline & Age & 0.125 & 0.724 & \\
\hline & Dose of SSRIs at T8 & 0.018 & 0.893 & \\
\hline & HAMD-17 at T0 & 0.254 & 0.614 & \\
\hline
\end{tabular}

SSRIs: Selective Serotonin Reuptake Inhibitors, BDNF: brain-derived neurotrophic factor, HAMD-17: 17-items of the Hamilton Rating Scale for Depression

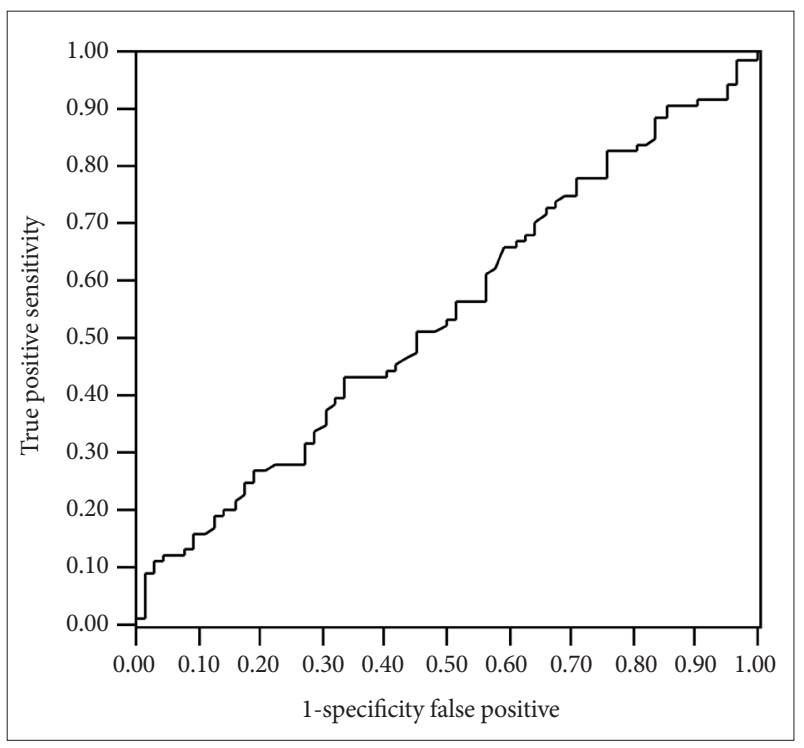

Figure 1. The ROC analysis of BDNF change from T0 to T4 for the response at T8. ROC: receiver operating characteristic, BDNF: brain-derived neurotrophic factor, T0: before staring SSRIs, T4: 4 weeks after SSRIs, T8: 8 weeks after SSRIs, SSRIs: Selective Serotonin Reuptake Inhibitors.

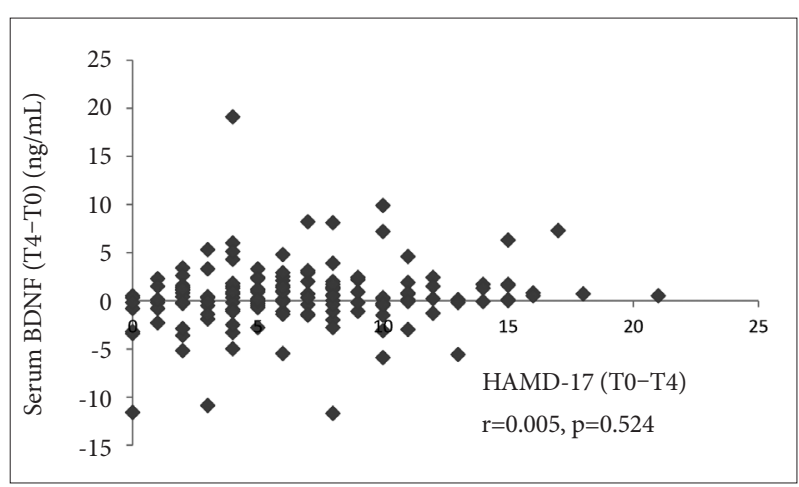

Figure 2. The changes in HAMD-17 (TO-4) and the changes in BDNF (T4-0). HAMD-17: Total scores in 17-items of Hamilton Rating Scale for Depression, T0: before staring SSRIs, T4: 4 weeks after SSRIs, SSRIs: Selective Serotonin Reuptake Inhibitors.

Any independent variables (changes in BDNF, sex, age, dose of SSRIs, and HAMD-17 at T0) were not associated with the response at T8 (Table 1). The AUC of a BDNF change from T0 to T4 was 0.54 for the response to at T8 ( $\mathrm{p}=0.311,95 \%$ confidence interval $=0.137-0.681$ ) (Figure 1 ). Significantly positive

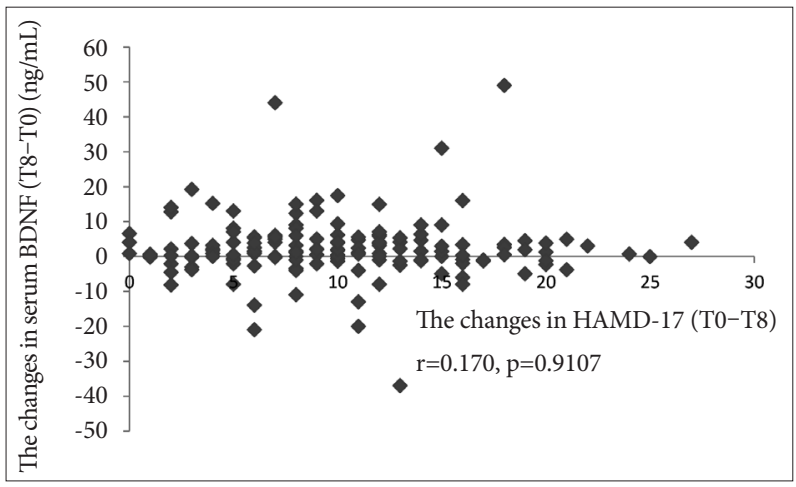

Figure 3. The changes in HAMD-17 (T0-8) and the changes in BDNF (T8-0). HAMD-17: Total scores in 17-items of Hamilton Rating Scale for Depression, T0: before staring SSRIs, T8: 8 weeks after SSRIs, SSRIs: Selective Serotonin Reuptake Inhibitors.

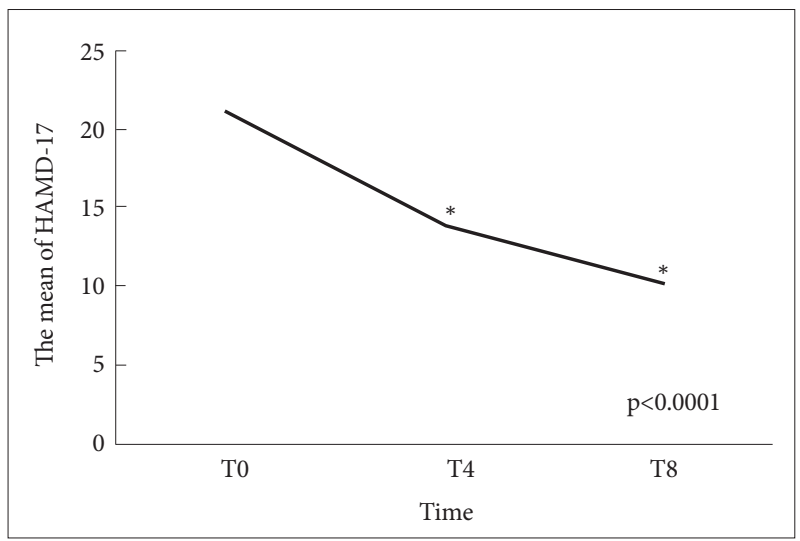

Figure 4. The changes in HAMD-17 at T0, T4, and T8. ${ }^{*} \mathrm{p}<0.0001$. HAMD-17: Total scores in 17-items of Hamilton Rating Scale for Depression, T0: before staring SSRIs, T4: 4 weeks after SSRIs, T8: 8 weeks after SSRIs, SSRIs: Selective Serotonin Reuptake Inhibitors.

correlations were not observed between the changes in HAMD-17 (T0-8) or (T0-4) and the changes in BDNF (T8-T0) or (T4-T0) ( $\mathrm{r}=0.005, \mathrm{p}=0.524)(\mathrm{r}=0.170, \mathrm{p}=0.9107)$ (Figures 2 and 3$)$. The scores of HAMD-17 significantly decreased from

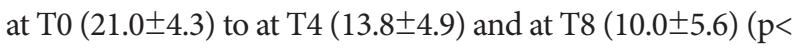
0.0001 ) (Figure 4). The serum BDNF levels significantly increased from at T0 $(8.6 \pm 7.2 \mathrm{ng} / \mathrm{mL})$ to at T8 $(11.2 \pm 8.4 \mathrm{ng} / \mathrm{mL})$, but not at $\mathrm{T} 4(9.2 \pm 6.1 \mathrm{ng} / \mathrm{mL})(\mathrm{p}=0.018)$ (Figure 5). 


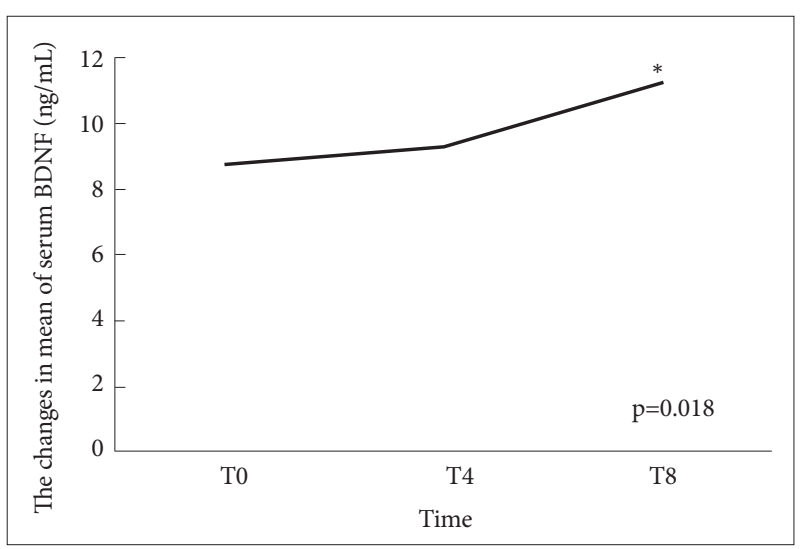

Figure 5. The changes in serum BDNF at T0, T4, and T8. ${ }^{*} p<0.05$. BDNF: brain-derived neurotrophic factor, TO: before staring SSRIs, T4: 4 weeks after SSRIs, T8: 8 weeks after SSRIs, SSRIs: Selective Serotonin Reuptake Inhibitors.

\section{DISCUSSION}

Treatment with SSRIs for 4 or 8 weeks significantly decreased the HAMD-17 scores in patients with MDD, and also increased serum BDNF levels at T8, but not at T4 (Figures 4 and 5).

In the present study, we demonstrated that changes in serum BDNF, sex, age, dose of SSRIs, and HAMD17 at baseline were not associated with the clinical response to SSRIs at day 28. The results in the present study revealed that the changes in serum BDNF and the HAMD-17 were both independent of the clinical outcome. Tadic et al. ${ }^{14}$ reported that the individual marker early serum BDNF non-increase has a positive predictive value for final treatment failure. In addition, Dreimuller et al. ${ }^{16}$ reported the changes in plasma BDNF at day 7 combined with HAMD improvement might be a predictor for good response to antidepressants. The between in the present study and those recent studies remain unknown. The early changes in BDNF only could not predict subsequently response to SSRIs. In other word, it might be necessary to combine the changes in HAMD scores. To the best of our knowledge, there have been at least four meta-analyses of studies of blood BDNF levels in depressed patients. ${ }^{6-9}$ The results of previous investigations have generally revealed that blood BDNF levels in depressed patients are significantly lower than those of healthy subjects. Taken together, these findings suggest that blood BDNF levels are a candidate biomarker for depression. However, it seems difficult to predict subsequent responses to SSRIs at early point of serum BDNF levels only. Actually, we previously reported that serum BDNF levels were not changed at all at T4, and were for the first time increased at T8. The results in the present study were not contradicted to our previous results in our previous study. ${ }^{11}$ In short, it takes at least 8 weeks or more to change the serum BDNF levels in patients with response to SSRIs. Furthermore, the AUC value in the present study was 0.54 , and the ROC was almost linear suggesting the prediction for response to SSRIs using the changes in serum BDNF levels (T0-T4)/T0 is low and not useful (Figure 1). This result was not contradicted with the results that no correlations were found the changes in HAMD (T0-4) or (T0-8) and the changes in BDNF (T4-0) or BDNF (T8-0).

There are several limitations in the present study. The source of circulating BDNF remains unknown. Platelets, brain, and vascular endothelial cells are considered candidate sources. BDNF assessed in serum represented the portion of BDNF stored in platelets, which bind, store, and release BDNF upon activation by a number factors, including physiological stress and injuries. But, even with agonist stimulation only about half of the BDNF in platelets is secreted, which suggests that platelets maintain a stable pool of BDNF, a buffer system. ${ }^{17}$ Previously, it was reported that BDNF levels in the brain and serum underwent similar changes during maturation and aging process in rats, ${ }^{18}$ indicating that plasma BDNF levels might in part reflect the BDNF in the brain. In contrast to this, Radka et al. ${ }^{19}$ reported that the BDNF detected in human serum was derived from platelet degranulation, and that circulating levels of BDNF are negligible. Furthermore, the plasma assay measures BDNF released in the blood, which is quite unstable. Plasma BDNF, for example, has very low retest stability when measured twice within one year, and there are substantial changes even during one day. Such variation is one drawback to assessing BDNF in plasma and may help explain the lack of association with measures of depression. Previous studies have also reported that a large degree of dispersion, which suggests that it is a problematic feature of the plasma BDNF measurement. A recent study also raises methodological concerns regarding the assessment of BDNF in plasma and recommends measuring BDNF in serum. ${ }^{17}$ From these findings into account, it is not clear as to what extent peripheral BDNF reflects central BDNF. Since it is well established that platelets are the major source of peripheral BDNF, ${ }^{17,21}$ it would be nice to have the BDNF data presented also in the framework of platelet counts of the investigated subjects. Furthermore, there is increasing evidence that sampling characteristics, several sociodemographic variables (such as urbanicity, age, sex), lifestyle factors (such as smoking status and food and alcohol intake), somatic diseases and even self-reported depressive symptoms are relevant determinants of peripheral BDNF levels. ${ }^{17-19}$ Thus, it is mandatory that the results presented are discussed with respect to these determinants. Therefore, further precise and well-controlled study considering above problems should be done to lead to robust results.

In conclusion, the changes in only serum BDNF levels from T0 to T4 could not predict the subsequent responses to SSRIs at $\mathrm{T} 8$. 


\section{REFERENCES}

1. Ruhe HG, Huyser J, Swinkels JA, Schene AH. Switching antidepressants after a first selective serotonin reuptake inhibitor in major depressive disorder: a systematic review. J Clin Psychiatry 2006;67:1836-1855.

2. Hamilton M. A rating scale for depression. J Neurol Neurosurg Psychiatry 1960;23:56-62.

3. Katz MM, Meyers AL, Prakash A, Gaynor PJ, Houston JP. Early symptom change prediction of remission in depression treatment. Psychopharmacol Bull 2009;42:94-107.

4. Martinowich K, Manji H, Lu B. New insights into BDNF function in depression and anxiety. Nat Neurosci 2007;10:1089-1093.

5. Duman RS, Heinger GR, Nestler EJ. A molecular and cellular theory of depression. Arch Gen Psychiatry 1997;54:597-606.

6. Sen D, Duman RS, Sanacora G. Serum brain-derived neurotrophic factor, depression, and antidepressant medications: meta-analyses and implications. Biol Psychiatry 2008;64:527-532.

7. Bocchio-Chiavetto L, Bagnardi V, Zanardini R, Molteni R, Nielsen MG, Placentino A, et al. Serum and plasma BDNF levels in major depression: a replication study and meta-analyses. World J Biol Psychiatry 2010;11: 763-773.

8. Brunoni AR, Lopes M, Fregni F. A systematic review and meta-analysis of clinical studies on major depression and BDNF levels: implications for the role of neuroplasticity in depression. Int J Neuropsychopharmmacol 2008;11:1169-1180.

9. Molendijk ML, Bus BA, Spinhoven P, Penninx BW, Kenis G, Prickaerts J, et al. Serum levels of brain-derived neurotrophic factor in major depressive disorder: state-trait issues, clinical features and pharmacological treatment. Mol Psychiatry 2010;16:1088-1095.

10. Katsuki A, Yoshimura R, Kishi T, Hori H, Umene-Nakano W, Ikenouchi-Sugita A, et al. Serum brain-derived neurotrophic factor (BDNF), BDNF gene Val66Met polymorphism, or plasma catecholamine metabolites, and response to mirtazapine in Japanese patients with major depressive disorder (MDD). CNS Spectr 2012;17:155-163.

11. Yoshimura R, Mitoma M, Sugita A, Hori H, Okamoto T, Umene W, et al. Effects of paroxetine or milnacipran on serum brain-derived neurotrophic factor in depressed patients. ProgNeuropsychopharmacol Biol Psychiatry 2007;31:1034-1037.
12. Umene-Nakano W, Yoshimura R, Ueda N, Suzuki A, Ikenouchi-Sugita A, Hori $\mathrm{H}$, et al. Predictive factors for responding to sertraline treatment: views from plasma catecholamine metabolites and serotonin transporter polymorphism. J Psychopharmacol 2010;24:1764-1771.

13. Wolkowitz OM, Wolf J, Shelly W, Rosser R, Burke HM, Lerner G, et al. Serum BDNF levels before treatment predict SSRI response in depression. Prog Neuropsychopharmacol Biol Psychiatyry 2011;35:1623-1630.

14. Tadic A, Wagner S, Schlicht KF, Peetz D, Boysenko L, Dreimuller N, et al. The early non-increase of serum BDNF predicts failure of antidepressant treatment in patients with major depression: a pilot study. Prog Neuropsychopharmacol Biol Psychiatyry 2011;35:415-420.

15. American Psychiatric Association. A Text Revision of Diagnostic and Statistical Manual of Mental Disorder. 4th Edition. Washington, DC: American Psychiatric Association; 2000.

16. Dreimuller N, Schlicht KF, Wagner S, Peetz D, Borysenko L, Hiemke C, et al. Early reactions of brain-derived neurotrophic factor in plasma (pBDNF) and outcome to acute antidepressant treatment in patients with major depression. Neuropharmacology 2012;62:264-269.

17. Bus BA, Molendijk ML, Penninx BJ, Buitelaar JK, Kenis G, Prickaerts J, et al. Determinations of serum brain-derived neurotrophic factor. Psychoendocrinology 2011;36:228-239.

18. Karege F, Schwald M, Cisse M. Postnatal developmental profile of brain-derived neurotrophic factor in rat brain and platelets. Neurosci Lett 2002;328:261-264.

19. Radka SF, Holst PA, Frische M, Alter CA. Presence of brain-derived neurotrophic factor in brain and human and rat but not mouse serum detected by sensitive and specific immunoassay. Brain Res 1996;709: 122-301.

20. Lommatzsch M, Zinger D, Schuhbaeck K, Schloetcke C, Zinger C, Schuff-Werner P, et al. The impact of age, weight and gender on BDNF levels in human platelets and plasma. Neurobiol Aging 2005;26:115123.

21. KaregeF, Bondolfi G, Gervason N, Schwald M, Aubry JM, Bertschy G. Low brain-derived neurotrophic factor (BDNF) levels in serum of depressed patients probably results from lowered platelet BDNF release unrelated to platelet reactivity. Biol Psychiatry 2005;57:1068-1072. 